\title{
Caracterización Fitoquímica y Efecto Hipoglucemiante de Tecoma stans y su Relación con la Presencia del Cromo como Factor de Tolerancia a la Glucosa
}

\author{
Ma. de J. Ibarra ${ }^{1}$, Pedro C. Cantú ${ }^{1}$, María J. Verde ${ }^{2}$ y Azucena Oranday ${ }^{2}$ \\ Universidad Autónoma de Nuevo León, (1) Facultad de Salud Pública y Nutrición y (2) Facultad de \\ Ciencias Biológicas, Av. Alfonso Reyes s/n, Cd. Universitaria, 64450 San Nicolás de los Garza, \\ Nuevo León, México (e-mail: mibarra@faspyn.uanl.mx, pcantu@faspyn.uanl.mx, \\ jverdestar@gmail.com, azucenaoranday@hotmail.com)
}

\begin{abstract}
Resumen
Se realizó la caracterización fitoquímica y la determinación del cromo en hojas de Tecoma stans, observando su efecto hipoglucemiante en animales con diabetes mellitus inducida en forma experimental. Como hiperglucemiante se utilizó estreptozotocina en ratas machos Sprague-Dawley a una dosis de $45 \mathrm{mg} / \mathrm{kg}$ de peso. Los tratamientos experimentales, todos por vía oral, fueron los siguientes para grupos diabéticos y grupos no diabéticos: agua, infusión de la planta, picolinato de cromo, e infusión combinada con picolinato de cromo. Se encontró cromo en $T$. stans $(54 \mu \mathrm{g} / 100 \mathrm{~g})$, la hiperglucemia disminuyó hasta $33.5 \%$ con $T$. stans, $10.1 \%$ con picolinato de cromo y $32.3 \%$ con la combinación de $T$. stans y picolinato de cromo, en 45 días. Con este estudio se confirma el efecto hipoglucemiante de $T$. stans.
\end{abstract}

\section{Phytochemical Characterization and Hypoglycemic Effect of the Tecoma stans and its Relationship to the Presence of Chromium as a Factor for Glucose Tolerance}

\begin{abstract}
Phytochemical characterization of Tecoma stans leaves, determination of chromium and their hypoglucemiant action in experimental animals with induced diabetes was done. The hyperglucemiant streptozotocin was employed in Sprague Dawley male rats at a dose of $45 \mathrm{mg} / \mathrm{kg}$. The experimental treatments, all orally delivered, were the following for both diabetic and non diabetic groups: water, infusion of the plant, chromium picolinate and infusion combined with chromium picolinato. Chromium was found in $T$. stans $(54 \mu \mathrm{g} / 100 \mathrm{~g})$, glucose levels decreased up to 33, $5 \%$ with $T$. stans, 10, $1 \%$ with chromium picolinate and $32,3 \%$ with the combination of $T$. stans and chromium picolinate, in 45 days. The hypoglucemiant effect of $T$. stans was confirmed with this study.
\end{abstract}

Keywords: tecoma stans, diabetes mellitus, chromium, glucose tolerance, phytochemistry 


\section{INTRODUCCIÓN}

La diabetes es una enfermedad crónica de gran importancia médicosocial porque afecta a muchos adultos y su frecuencia está aumentando con el envejecimiento de las poblaciones. A nivel mundial, en el año 2000 sufrían diabetes 171 millones de personas, y se prevé que en el 2030 esa cifra alcance un total de 366 millones (Kalofoutis et al., 2007). En algunos países, 1 de cada 20 personas sufre diabetes, pero menos de la mitad de los casos son diagnosticados. En México, aproximadamente el $10 \%$ de la población padece diabetes, se estima que el $90 \%$ de los casos son del tipo II. En nuestro país, la diabetes es una enfermedad bastante frecuente entre los ancianos y entre las personas obesas, no es una enfermedad contagiosa, siendo la principal causa de mortandad, y causando el 12\% de las muertes (Forga y Zulet, 2002; Cabrera et al., 2008).

Diversos estudios se han reportado en donde se refleja la actividad biológica de productos vegetales; como ejemplo se puede citar el trabajo de Murillo et al. (2007), en donde se demuestra la actividad antioxidante de los extractos de Bauhinia kalbreyeri Harms, conocida también como antidiabética. Otro estudio realizado por Arriola et al. (2006), reporta actividad germicida en la semilla del limón mexicano (Citrus aurantifolia swingle). Investigaciones etnobotánicas realizadas en México, reportan que la población utiliza de manera empírica entre 150 y 269 especies de plantas para el control de la diabetes mellitus. Algunos estudios sugieren que es necesario un estudio más exhaustivo de este tipo de plantas medicinales, para poder demostrar su utilidad en el manejo de la diabetes mellitus no insulinodependiente (Alarcón et al., 1993; Hernández et al., 2002; Prabhakar y Doble, 2008). Entre las plantas medicinales del noreste de México, se ha encontrado que algunas de éstas son utilizadas por las personas como antidiabéticas. Opuntia streptacantha y Tecoma stans son las plantas mexicanas más utilizadas como antidiabéticas y son también las plantas hipoglicémicas mas estudiadas en el tratamiento del efecto hipoglucemiante (González, 1998; Adame y Adame, 2000; Naranjo et al., 2003). Además, es importante intensificar los estudios químicos y farmacológicos de las plantas con este efecto porque solo pocos agentes hipoglicémicos han sido aislados y caracterizados químicamente (Hernández et al., 2002; Andrade y Henrich, 2005).

Se ha encontrado que el mineral cromo, que en la actualidad se utiliza en algunos productos dietéticos en forma de picolinato de cromo, ha demostrado tener efectos benéficos en diversos estudios, sobre todo, en aquellos relacionados con la diabetes y el metabolismo del azúcar; estos estudios comprueban que el cromo es un factor primordial para el buen metabolismo del azúcar sanguíneo (Anderson, 1998; Broadhurst y Domenico, 2006; Guerrero y Rodríguez, 2005).

Por esta razón se planteó realizar la evaluación fitoquímica de $T$. stans (L) Juss. Ex. Kunt (tronadora) considerada con efecto hipoglucemiante; la cual se encuentra distribuida en el noreste de México; relacionando esta identificación con la presencia del elemento cromo como factor de tolerancia a la glucosa y observando el efecto hipoglucemiante de los extractos acuosos de estas plantas en modelos de ratas con diabetes inducida experimentalmente.

\section{METODOLOGÍA}

Área de recolección, muestreo e identificación de la especie

Durante la época de verano se recolectó $T$. stans en el municipio de Los Rayones, Nuevo León, México, siendo las coordenadas de la cabecera municipal de Rayones: latitud norte $25^{\circ}$, longitud oeste $100^{\circ}$ 04', mientras que la altitud es de 906 metros sobre el nivel del mar. Se seleccionaron especímenes sanos, maduros y sin daño aparente, siguiendo un procedimiento aleatorio simple. Se llevó un ejemplar seco de la planta al Departamento de Botánica de la Facultad de Ciencias Biológicas, UANL. para su identificación sistemática.

\section{Molienda, secado y obtención de extracto}

Se separaron las hojas de $T$. stans, se secaron a temperatura ambiente y con aireación por una semana y se molieron en molino eléctrico, utilizando un tamaño de malla mediano. Para obtener el extracto acuoso se pesaron 50 gramos de planta seca y molida y se preparó una infusión en agua, se reposó por 20 minutos y se filtró utilizando papel filtro Whatman No. 40 en matraz kitasato y con la 
ayuda de una bomba de vacío. El sobrenadante se colocó en viales especiales para liofilizadora, se congelaron a $-21^{\circ} \mathrm{C}$ por 12 horas y después a $-70^{\circ} \mathrm{C}$ para su posterior liofilización (LABCONCO, Freeze Dry System, Freezone 12). Para la obtención del extracto hexánico se colocaron en un matraz Erlenmeyer 50 gramos de la planta seca y molida, se agregaron $250 \mathrm{~mL}$ de hexano, se tapó el matraz y se colocó en un agitador eléctrico por 48 horas, se filtró como se explicó anteriormente para el extracto acuoso. El sobrenadante se eliminó por evaporación a $37^{\circ} \mathrm{C}$ por 3 días y se obtuvo el extracto hexánico, se pesó el rendimiento. Para la obtención del extracto etanólico se colocó en un matraz Erlenmeyer el sólido restante del extracto anterior con $250 \mathrm{~mL}$ de etanol absoluto, se tapó el matraz y se colocó en un agitador eléctrico por 72 horas, se filtró de la misma forma que para el extracto anterior. Se desechó la parte sólida y el sobrenadante se concentró bajo las mismas condiciones que el extracto hexánico, se obtuvo el extracto etanólico y se pesó el rendimiento.

\section{Pruebas fitoquímicas y Cromatografía en capa fina (CCF)}

Una porción de cada uno de los extractos fue disuelta en agua, hexano y/o etanol y de esta mezcla se tomó una muestra para el análisis fitoquímico, siguiendo los procedimientos reportados por Domínguez (1979). Los metabolitos que se analizaron fueron alcaloides (test de Wagner), coumarinas $(\mathrm{NaOH})$, flavonoides (test de Salkowski), sesquiterpenlactonas (test de Baljet), esteroles, metilesteroles y saponinas (test de Liebermann-Burchard), azúcares (test de Molish), quinonas (test de Bôrntrager) e insaturaciones $\left(\mathrm{KMnO}_{4}\right)$. Posteriormente se realizó el corrimiento de cada extracto mediante CCF, para la identificación de los alcaloides, se revelaron las placas cromatográficas con el reactivo de Dragendorff.

\section{Determinación del cromo}

El cromo se determinó en 100 gramos de la planta seca y molida por espectrofotometría de absorción atómica (Perkin-Elmer 5100) siguiendo el procedimiento marcado por la norma oficial NMX-AA-051-SCFI (2001).

\section{Dosis del hiperglucemiante}

Para determinar la dosis óptima del hiperglucemiante se utilizaron 25, 35 y $45 \mathrm{mg} / \mathrm{kg}$ de peso de estreptozotocina (EZT) (Sigma) administrado por vía intraperitoneal en ratas Sprague-Dawley, monitoreando la concentración de glucosa sanguínea hasta alcanzar una diabetes moderada (170$300 \mathrm{mg} / \mathrm{dL}$ de glucosa).

\section{Planteamiento experimental}

Para conocer el efecto antidiabético de $T$. stans se utilizaron 24 animales de laboratorio, de los cuales a 12 se les indujo la diabetes, de tal forma que se tuvieron 8 grupos $(n=3)$ y diariamente se administró lo siguiente:

*Grupo diabético (n=3): agua por vía oral (v.o)

*Grupo diabético $(n=3)$ : infusión de la planta por v.o.

${ }^{*}$ Grupo diabético $(\mathrm{n}=3)$ : picolinato de cromo $(200 \mu \mathrm{g} / \mathrm{kg}$ de peso) por v.o.

*Grupo diabético $(n=3)$ : picolinato de cromo más infusión de la planta por v.o.

*Grupo no diabético $(n=3)$ : agua por v.o

*Grupo no diabético $(n=3)$ : infusión de la planta por v.o.

*Grupo no diabético $(n=3)$ : picolinato de cromo por v.o.

*Grupo no diabético $(n=3)$ : picolinato de cromo más infusión de la planta por v.o.

Se les realizó seguimiento de peso, niveles de glucosa sanguínea y urinaria; así como se observó el comportamiento renal y hepático. La determinación de los niveles de glucosa se realizó a los 7, 15, 30 y 45 días, después de administrado el EZT y el extracto de la planta. Para la glucosa sanguínea se tuvo un ayuno de 4-5 horas en cada uno de los animales, se obtuvo sangre de la cola, se utilizaron tiras reactivas y un glucómetro manual (Roche). Para la determinación de la glucosa urinaria se tuvo un ayuno previo de 7 horas, se colectó orina de los animales y se utilizaron tirillas reactivas especiales para uroanálisis. Para el análisis estadístico, se utilizó la prueba de ANOVA de una sola vía. 


\section{RESULTADOS Y DISCUSIÓN}

\section{Caracterización fitoquímica}

El rendimiento de los extractos fue: acuoso 16.35 g., 2.1 g. para el hexánico y $6.27 \mathrm{~g}$. para el etanólico. La tabla 1 muestra los resultados del análisis fitoquímico en los extractos de $T$. stans. En los tres extractos se encontró presencia de: alcaloides, coumarinas, flavonoides y sesquiterpenlactonas. Encontramos esteroles, metilesteroles y saponinas en los extractos hexánico y etanólico. Se observaron insaturaciones para los extractos acuoso y etanólico. Mientras que los azúcares solo se encontraron presentes en el extracto acuoso.

Tabla 1: Análisis fitoquímico en los extractos de las hojas de Tecoma stans

\begin{tabular}{|c|c|c|c|c|}
\cline { 3 - 5 } \multicolumn{2}{c}{} & \multicolumn{3}{c|}{ Ex t r a c t o s } \\
\hline Componentes & Prueba & Acuoso & Etanólico & Hexánico \\
\hline Alcaloides & Wagner & + & + & + \\
\hline Coumarinas & NaOH & + & + & + \\
\hline Flavonoides & Salkowski & + & + & + \\
\hline Sesquiterpenlactonas & Baljet & + & + & + \\
\hline $\begin{array}{c}\text { Esteroles y } \\
\text { metilesteroles }\end{array}$ & $\begin{array}{c}\text { Liebermann- } \\
\text { Burchard }\end{array}$ & - & + & + \\
\hline Azúcares & Molish & + & - & - \\
\hline Saponinas & $\begin{array}{c}\text { Liebermann- } \\
\text { Burchard }\end{array}$ & - & + & + \\
\hline Quinonas & Borntrager & - & + & - \\
\hline Insaturaciones & KMnO $_{4}$ & + & + & - \\
\hline
\end{tabular}

\section{Eluentes probados para cromatografía en capa fina (CCF) y presencia de alcaloides}

En cuanto a los eluentes probados para cada extracto de $T$. stans, el eluente que mostró mejor separación de bandas para el extracto acuoso fue cloroformo:metanol:HOAC (65:35:10), para el extracto etanólico cloroformo:metanol:agua (65:35:10) y para el extracto hexánico fue éter de petróleo:benceno:acetona (9:9:2) y estos eluentes fueron utilizados para realizar la identificación de alcaloides por CCF. En la tabla 2 se observa el tipo de eluente utilizado en cada extracto, así como el número, color de bandas y relación de frente (RF) al revelar con luz ultravioleta (UV).

Tabla 2: Cromatografía de cada extracto y revelación de alcaloides

\begin{tabular}{|c|c|c|c|}
\hline Extracto & Eluente & $\begin{array}{l}\text { No. de bandas } \\
\text { (UV) y }{ }^{* \star} \text { RF }\end{array}$ & $\begin{array}{l}\text { Resultado para } \\
\text { alcaloides } \\
\text { (Dragendorff) }\end{array}$ \\
\hline Acuoso & $\begin{array}{c}\text { Cloroformo:Metanol:HOAC } \\
(65: 35: 10)\end{array}$ & $\begin{array}{ll}1 & 0.55 \\
2 & 0.75 \\
3 & 0.85\end{array}$ & Positivo \\
\hline Etanólico & $\begin{array}{l}\text { Cloroformo:Metanol:Agua } \\
\qquad(65: 35: 10)\end{array}$ & $\begin{array}{ll}1 & 0.31 \\
2 & 0.56 \\
3 & 0.88 \\
4 & 0.95 \\
\end{array}$ & Positivo \\
\hline Hexánico & $\begin{array}{c}\text { Eter de } \\
\text { petróleo:benceno:Acetona } \\
(9: 9: 2)\end{array}$ & $\begin{array}{ll}1 & 0.13 \\
2 & 0.38 \\
3 & 0.48 \\
4 & 0.63\end{array}$ & Positivo \\
\hline
\end{tabular}

Se encontró la presencia de 3, 4 y 4 bandas respectivamente y al revelar con Dragendorff dio positivo para alcaloides en los 3 extractos. El hecho de encontrar presencia de alcaloides en los tres extractos concuerda con dos trabajos reportados por Constantino et al. (2003a y 2003b), en donde 
estudia el aislamiento y caracterización de las actividades farmacológicas de alcaloides de $T$. stans encontrando que los alcaloides tecomanina y tecostatina mostraron ser responsables del efecto hipoglicémico. Además, Hammouda et al. (1964) y Hammouda y Amer (1966), demostraron también que la tecostatina y tecomanina administradas intravenosamente producen un efecto hipoglucémico más fuerte que el inducido por tolbutamida en conejos.

\section{Concentración de cromo}

Se encontró cromo en las hojas de $T$. stans en una concentración de $54 \mu \mathrm{g} / 100 \mathrm{~g}$. Como se mencionó anteriormente, el cromo se considera elemento traza esencial en humanos y animales y tiene una función importante en el metabolismo de la insulina, como factor de tolerancia a la glucosa (FTG). El hecho de encontrarlo presente en esta especie resulta favorable en la reducción de la hiperglicemia (Anderson 1998). Múltiples estudios se han realizado para estudiar la función del cromo, como factor de tolerancia a la glucosa (Chowdhury et al., 2003; Brown 2003; Broadhurst y Domenico, 2006; Balk et al., 2007), sin embargo, solo se encuentra un trabajo reportado (Castro et al., 2002) y el presente estudio en el que se asocia este elemento y su efecto hipoglucemiante.

\section{Concentración de EZT y producción de modelos diabéticos}

La dosis de EZT que produjo diabetes moderada (170-300 $\mathrm{mg} / \mathrm{dL}$ ) fue la de $45 \mathrm{mg} / \mathrm{kg}$ de peso a los 7 días después de aplicar una segunda dosis; obteniendo una concentración promedio de glucosa sanguínea de $265 \pm 33.8 \mathrm{mg} / \mathrm{dL}$.

\section{Identificación de la concentración de Tecoma stans para reducción de la hiperglucemia}

Una vez que se identificó la dosis de EZT (45 mg/kg de peso) que logró producir la hiperglucemia, se procedió a la identificación de la dosis de infusión acuosa de $T$. stans a la cual se presentara reducción de la hiperglucemia. Se utilizaron concentraciones de manera progresiva $(0.6,1.6,5$ y 25 $\mathrm{g} / \mathrm{L}$ ) de infusión acuosa de la planta. Encontrando que con $25 \mathrm{~g} / \mathrm{L}$ se observó una disminución del 10 $\%$ de la hiperglucemia a los 7 días de tratamiento. Para el bioensayo definitivo se utilizó una concentración de infusión de $T$. stans de $30 \mathrm{~g} / \mathrm{L}$.

La figura 1 muestra que el grupo de animales diabéticos que recibió la infusión de Tecoma stans se redujo un $33.5 \%$ la hiperglucemia, en el grupo tratado con picolinato de cromo un $10.1 \%$ y la mezcla de $T$. stans con picolinato de cromo redujo un $32.3 \%$ a los 45 días después de administrar los diferentes tratamientos. Al comparar los diferentes tratamientos en estos grupos, encontramos que existe una diferencia estadísticamente significativa con respecto al grupo control $(F=8.76, p<0.05)$ (ANOVA). Mientras que al aplicar los tratamientos en los grupos de animales no diabéticos no se presentaron cambios en cuanto a la concentración de glucosa (figura 2).

Aunque se conoce que el cromo favorece la reducción de la hiperglucemia, el grupo tratado con la planta más picolinato de cromo tuvo menor porcentaje de disminución de la hiperglucemia con respecto al grupo tratado solamente con $T$. stans $(32.3 \%$ vs $33.5 \%)$, sin mostrar diferencia significativa ( $p>0.05$ ); posiblemente esto se deba a la cantidad de cromo utilizada (equivalente a 200 $\mu \mathrm{g} / \mathrm{día}$ ). La literatura reporta cantidades recomendadas de cromo en la dieta que van desde 50 a 200 $\mu \mathrm{g}$ por día para personas adultas (Caffaratti y Briñón, 2005); aunque existen estudios reportados en sujetos diabéticos donde se utilizan cantidades mayores a las recomendadas. Un estudio descubrió que los diabéticos que tomaron 1,000 microgramos de cromo diario experimentaron una reducción significativa de niveles de azúcar en la sangre (Balk et al., 2007). Posiblemente, para observar una disminución significativa de la hiperglucemia en el grupo tratado con la planta más picolinato de cromo, en el presente trabajo se debieron utilizar dosis mayores de picolinato de cromo, puesto que los animales estudiados eran diabéticos.

Los resultados obtenidos en el presente estudio sobre el efecto hipoglucemiante de $T$.stans, se relacionan con trabajos de Lozoya (1980) y Lozoya y Mellado (1985), quienes reportaron efecto hipoglucémico después de la administración oral de infusión de $T$. stans en conejos y perros, respectivamente. Posteriormente, Bnouham (2006) en un estudio de revisión sobre plantas 
medicinales con potencial actividad antidiabética menciona que $T$. stans disminuye significativamente el área bajo la curva de tolerancia a la glucosa con respecto al grupo control $(17.5 \%)$ o tolbutamida (14.3\%) en conejos a los cuales se les administró gástricamente agua, tolbutamida o la preparación de la planta

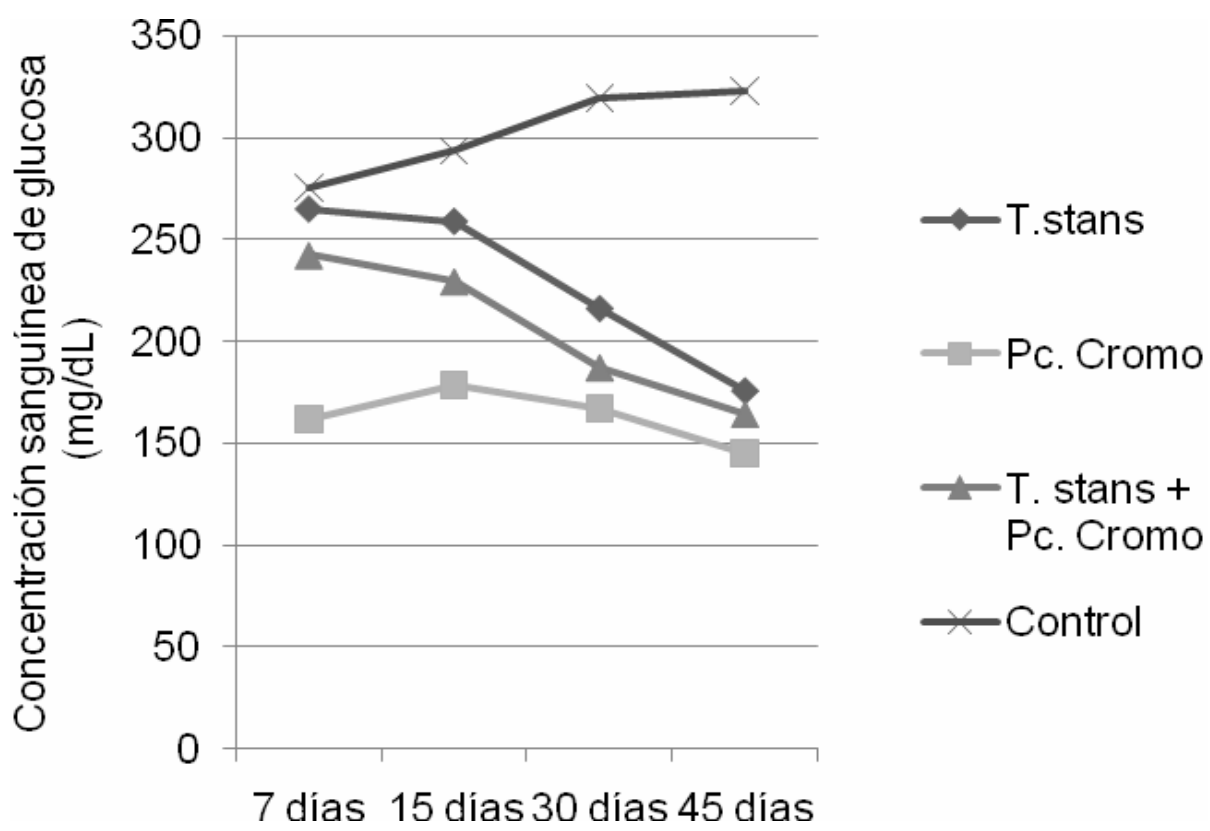

Fig.1: Comportamiento de la concentración de glucosa sanguínea en animales diabéticos durante el tiempo de tratamiento.

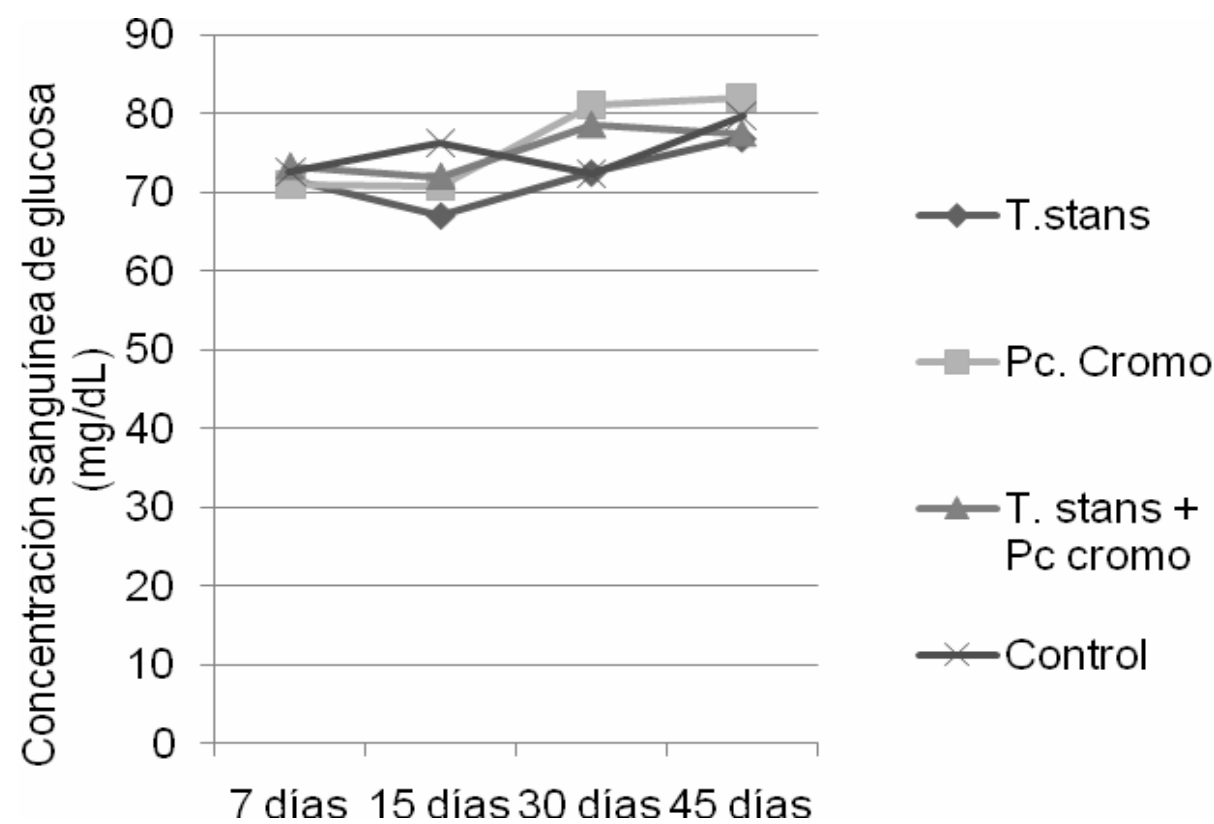

Fig. 2: Comportamiento de la concentración de glucosa sanguínea en animales no diabéticos durante el tiempo de tratamiento.

Los resultados obtenidos no son exactamente comparables con otras investigaciones en donde se estudia el efecto hipoglucémico de esta planta debido a que existen variaciones en cuanto al modelo animal utilizado, vía de administración, dosis de la planta y el modo de preparación del extracto. 
Resultados de glucosa urinaria, comportamiento renal, hepático y equilibrio ácido-básico.

Las tablas 4 y 5 muestran los resultados del uroanálisis en los grupos de animales diabéticos, en los cuales, a los 45 días de tratamiento en estos animales se encontró trazas de leucocitos y presencia de proteínas en los 4 grupos tratados. La diabetes mellitus (DM) es un factor predisponente para adquirir infección en vías urinarias (IVU). Las IVU se relacionan con la descompensación y elevación de la glicemia, pueden evolucionar hacia la cronicidad $\mathrm{y}$, por tanto, promover fallo renal $\mathrm{y}$ consecuentemente un deterioro de la calidad de vida de los pacientes que la padecen (Flores et al., 2005).

La presencia de trazas de leucocitos en casi todos los grupos de animales diabéticos en los diferentes tratamientos, es un signo claro de inflamación a nivel renal y de las vías urinarias. Las IVU se encuentran relacionadas con problemas obstructivos y alteraciones en la función del tracto urinario (Morrison, 1998). Los pacientes diabéticos tienen dos veces más riesgo de adquirir infecciones complicadas del tracto urinario en comparación con los que no la padecen; la pielonefritis aguda es cinco veces más frecuente en diabéticos; en $60 \%$ de los pacientes hospitalizados con bacteremia y diabetes, la fuente de infección son las vías urinarias (Flores et al., 2005).

La presencia de proteínas en la orina de la gran mayoría de los grupos de animales diabéticos en los diferentes tratamientos pudo deberse a una complicación renal conocida como nefropatía diabética (ND), durante la cual el riñón presenta daño y se acumula mas proteína en la orina de lo normal. A medida que la enfermedad progresa cada vez se destruye mas parte del riñón y con el tiempo la capacidad de este para funcionar comienza a declinar, lo que finalmente puede llevar a insuficiencia renal crónica (IRC).

Tabla 4: Valores promedio de los indicadores del examen general de orina (EGO) en los grupos de animales diabéticos a los 7 días después de administrar los diferentes tratamientos.

\begin{tabular}{|c|c|c|c|c|c|}
\hline Indicador & $\begin{array}{c}\text { basal } \\
(\mathrm{n}=20)\end{array}$ & $\begin{array}{c}\text { T. stans } \\
(\mathrm{n}=3)\end{array}$ & $\begin{array}{c}\text { Picolinato } \\
\text { de cromo } \\
(\mathrm{n}=3)\end{array}$ & $\begin{array}{c}\text { T. stans y } \\
\text { picolinato } \\
\text { de cromo } \\
(\mathrm{n}=3)\end{array}$ & $\begin{array}{c}\text { Control } \\
\text { diabético } \\
(\mathrm{n}=3)\end{array}$ \\
\hline Leucocitos & - & - & Trazas & Trazas & Trazas \\
\hline Nitritos & - & + & - & - & - \\
\hline Urobilinógeno & Normal & Normal & Normal & Normal & Normal \\
\hline Proteínas & - & +++ & ++++ & +++ & +++ \\
\hline $\mathrm{pH}$ & 7.0 & 8.0 & 8.0 & 7.5 & 7.25 \\
\hline Sangre & - & - & - & - & - \\
\hline Densidad (g/mL) & 1.005 & 1.015 & 1.005 & 1.008 & 1.003 \\
\hline Cetonas & - & - & - & - & - \\
\hline Bilirrubina & - & - & - & - & - \\
\hline Glucosa (mg\%) & - & 150 & - & 150 & 250 \\
\hline
\end{tabular}

La presencia de cantidades altas de glucosa puede dañar las estructuras del riñón, haciendo que se vuelvan gruesas y cicatricen. Lentamente con el tiempo más y más vasos sanguíneos resultan destruidos. Las estructuras renales se empiezan a filtrar y la proteína (albúmina) empieza a salir en la orina (McPherson et al., 2006; Parving et al., 2007). Además, se encontró presencia de glucosa en el grupo tratado con $T$. stans y en el grupo control. En general, la glucosa no aparece en orina hasta que su concentración plasmática no sobrepasa la capacidad de reabsorción tubular (aproximadamente $180 \mathrm{mg} / \mathrm{dL}$ ). No obstante, en algunas personas sanas la glucosa puede pasar a la orina a concentraciones mucho menores. Por el contrario, el umbral renal de la glucosa aumenta con la edad y como consecuencia, muchos diabéticos no excretan glucosa en la orina. Es importante recordar que la concentración de glucosa urinaria es el reflejo de la concentración plasmática durante el tiempo de formación de la orina, por lo que no refleja la glucemia en el momento del examen de la 
muestra de orina. La presencia de glucosuria posee una elevada sensibilidad para el diagnóstico de diabetes mellitus, por lo que la determinación de glucosa en orina puede ser un parámetro de cribado de esta enfermedad (Flores et al., 2005; ADA, 2008). En los grupos de animales no diabéticos tratados no se obtuvo la presencia o valores anormales de los indicadores analizados en el uroanálisis.

Tabla 5: Valores promedio de los indicadores del EGO en los grupos de animales diabéticos a los 45 días después de administrar los diferentes tratamientos.

\begin{tabular}{|c|c|c|c|c|}
\hline Indicador & $\begin{array}{c}\text { T. stans } \\
(\mathrm{n}=3)\end{array}$ & $\begin{array}{c}\text { Picolinato } \\
\text { de cromo } \\
(\mathrm{n}=3)\end{array}$ & $\begin{array}{c}\text { T. stans y } \\
\text { picolinato de } \\
\text { cromo }(\mathrm{n}=3)\end{array}$ & $\begin{array}{c}\text { Control } \\
\text { diabético } \\
(\mathrm{n}=3)\end{array}$ \\
\hline Leucocitos & Trazas & Trazas & Trazas & Trazas \\
\hline Nitritos & + & - & - & + \\
\hline Urobilinógeno & Normal & Normal & Normal & Normal \\
\hline Proteínas & +++ & ++++ & +++ & +++ \\
\hline pH & 7.0 & 7.5 & 7.5 & 7.25 \\
\hline Sangre & - & - & - & + \\
\hline Densidad $(\mathrm{g} / \mathrm{mL})$ & 1.020 & 1.015 & 1.005 & 1.010 \\
\hline Cetonas & - & - & - & + \\
\hline Bilirrubina & - & - & - & - \\
\hline Glucosa $(\mathrm{mg} \%)$ & 100 & - & - & 350 \\
\hline
\end{tabular}

\section{CONCLUSIONES}

En los grupos de animales diabéticos a los cuales se les administraron los diferentes tratamientos encontramos una disminución de la hiperglucemia de manera significativa con respecto al grupo control diabético $(p<0.05)$. Observamos que a los 45 días de tratamiento, el grupo al cual se le administró la infusión de $T$. stans fue el que mostró el mayor porcentaje de reducción de la hiperglucemia (33.5\%). Aunque se conoce que el cromo favorece la reducción de la hiperglucemia, el grupo tratado con la planta mas picolinato de cromo tuvo menor porcentaje de disminución de la hiperglucemia con respecto al grupo tratado solamente con $T$. stans (32.3\% vs 33.5\%), sin mostrar diferencia estadísticamente significativa entre estos dos grupos $(p>0.05)$. Al aplicar los tratamientos en los grupos de animales no diabéticos no se presentaron cambios en cuanto a la concentración de glucosa. En la caracterización fitoquímica, el extracto etanólico fue el que mostró el mayor número de familias de metabolitos. Se identificó la presencia de alcaloides en los 3 extractos de la planta. Al final de los diferentes tratamientos, todos los grupos diabéticos presentaron leucocitos y proteínas en orina, lo que indica una infección, así como probable daño en la función glomerular. Con este estudio se confirma que $T$. stans posee efecto hipoglucemiante.

\section{AGRADECIMIENTOS}

Al Consejo Nacional de Ciencia y Tecnología (CONACYT) por el apoyo financiero otorgado. A QFB. Jorge Galván Solís, Dra. Catalina Rivas Morales y MVZ. Ramón Belmontes Hernández por su colaboración en el presente trabajo.

\section{REFERENCIAS}

Adame J. y H. Adame; Plantas curativas del Noreste Mexicano, 1a. edición, 1-386. Ed. Castillo. México (2000).

Alarcón F.J., R. Román y J.L. Flores; Plantas medicinales usadas en el control de la diabetes mellitus, Ciencia: 44(3), 363-381 (1993).

ADA, American Diabetes Association; Standards of Medical Care in Diabetes, Diabetes Care: 31, S12-54S (2008). 
Anderson R.A.; Chromium, glucosa intolerante and diabetes, J Am Coll Nutr: 17, 548-555 (1998).

Andrade C.A. y M. Henrich; Mexican plants with hypoglycaemic effect used in treatment of diabetes, J Ethnopharmacol.: 99(3), 325-348 (2005).

Arriola E., T. García, G.M. Guatemala, J.A García; Estudio preliminar de las propiedades de la semilla de limón mexicano (Citrus aurantifolia swingle) para su posible aprovechamiento, Información Tecnológica: 17(6), 97-102 (2006).

Balk E.M., A. Tatsioni, A.H. Lichtenstein, J. Lau y A.G. Pittas; Effect of chromium supplementation on glucose metabolism and lipids: a systematic review of randomized controlled trials, Diabetes Care: 30(8), 2154-2163 (2007).

Bnouham M., A. Ziyyat y H. Mekhfi; Medicinal plants with potential antidiabetic activity -A review of ten years of herbal medicine research, Int J Diabetes \& Metabolism: 14, 1-25 (2006).

Broadhurst C.L. y P. Domenico; Clinical studies on chromium picolinato supplementation in diabetes mellitus -a review, Diabetes Technol. Ther.: 8(6), 677-687 (2006).

Brown M.; Hernessing chromium in the figth against diabetes, Drug Discov. Today: 8, 962-963 (2003).

Cabrera C. y otros seis autores; Family physicians clinical aptitude for the nutritional management of type 2 diabetes mellitus in Guadalajara, Mexico, J Nutr Health Aging: 12, 156-160 (2008).

Caffaratti M. y M.C. Briñón; Suplementos dietarios: Picolinato de cromo (Revisión), Centro de Información de Medicamentos (CIME) (2005), http://www.fcq.unc.edu.ar/cime/picolinatodecromo.htm. Acceso: 9 de Enero (2009).

Castro A. y otros nueve autores; Investigación de metabolitos secundarios en plantas medicinales con efecto hipoglucemiante y determinación del cromo como factor de tolerancia a la glucosa, Ciencia e Investigación: 5, 3-6 (2002).

Constantino L., y otros nueve autores; Isolation and pharmacological activities of the Tecoma stans alkaloids, Farmaco: 58(9), 781-5 (2003a).

Constantino L., y otros siete autores; Characterization and pharmacological actions of tecostatine, an alkaloid of Tecoma stans, Pharmazie: 58(2), 140-2 (2003b).

Chowdhury S., K. Pandit., P. Roychowdury, B. Bhattacharya; Role of chromium in human metabolism, with special reference to type 2 diabetes, J. Assoc Physicians India: 51, 701-705 (2003).

Domínguez X.A.; Métodos de investigación fitoquímica, 1a. edición, 1-281. Ed. Limusa, México (1979).

Flores E., I. Parra, A. Jiménez y G. Fernández; Pruebas presuntivas del análisis de orina en el diagnóstico de infección en vías urinarias entre diabéticos tipo 2, Salud Pública Méx.: 47, 376-380 (2005).

Forga L. y A. Zulet; Alimentación y Salud Pública, En: Alimentación y Salud Pública por J.A. Martínez, I. Astiasarán y H. Madrigal, 2aa. Edición, Editorial Mc. Graw Hill, 198-205, México (2000).

González F.M.; Plantas Medicinales del Noreste de México, 1ª Edición, 1-128. IMSS, México (1998).

Guerrero F. y M. Rodríguez; Complementary Therapies for diabetes: the case for chromium, magnesium, and antioxidants, Arch. Med. Res.: 36(3), 250-257 (2005). 
Hammouda Y., A. Rashid y M. Amer; Hypoglycemic properties of tecomine and tecostatine, Journal of Pharmacy and Pharmacology: 16, 833-834 (1964).

Hammouda Y. y M. Amer; Antidiabetic effect of tecomine and tecostatine, Journal of Pharmaceutical Science: 55, 1452-1454 (1966).

Hernández G.E. y otros siete autores; Studies on hypoglycemic activity of mexican medicinal plants, Proc. West. Pharmacol. Soc.: 45, 118-124 (2002).

Kalofoutis C. y otros cinco autores; Type II diabetes mellitus and cardiovascular risk factors: Current therapeutic approaches, Exp Clin Cardiol: 12, 17-28 (2007).

Lozoya M.; Tronadora (Tecoma stans L. H.B.K.), Medicina tradicional (México): 3, 5-9 (1980).

Lozoya M. y C. Mellado; Is the Tecoma stans infusion an antidiabetic remedy? Journal of Ethnopharmacology: 14, 1-9 (1985).

McPherson R.A., J. Ben-Ezra y S. Zhao; Basic examination of urine. In: McPherson \&Pincus: Henri's Clinical Diagnosis and Management by Laboratory Methods by McPherson RA, Pincus MR. $21^{\text {st }}$ ed. Pa: WB. Saunders Company, chap.27. Philadelphia (2006).

Morrison K.; Laboratorio Clínico y Pruebas de Diagnóstico, 1ª Edición, 97-106. El Manual Moderno, México (1998).

Murillo E., O. Lombo, M. Tique y J.J. Méndez; Potencial antioxidante de Bauhinia kalbreyeri Harms (FABACEAE), Información Tecnológica: (18)6, 65-74 (2007).

Naranjo J., A. Corral, G. Rivero, M. Fernández y P. Pérez; Efecto hipoglicemiante del extracto fluido de Tecoma stans Linn en roedores, Rev. Cubana Milit.: 32(1), 13-17 (2003).

NMX-AA-051-SCFI-2001; Norma Oficial Mexicana sobre Análisis de agua - Determinación de metales por absorción atómica en aguas naturales, potables, residuales y residuales tratadas Método de prueba, 1-52, México, D.F. (2001).

Parving H., M. Mauer y E. Ritz; Diabetic Nephropathy. In: Brenner and Rector's the Kidney by Brenner BM. 8th ed. Pa: Saunders Elsevier, chap 36. Philadelphia (2007).

Prabhakar P.K, y M. Doble; A target based therapeutic approach towards diabetes mellitus using medicinal plants, Curr Diabetes Rev: 4(4), 291-308 (2008). 